\title{
High density lipoprotein is positively correlated with the changes in circulating total adiponectin and high molecular weight adiponectin during dietary and fenofibrate treatment
}

\author{
Georgios A. Christou, ${ }^{1}$ Constantinos C. Tellis, ${ }^{2}$ Moses S. Elisaf, ${ }^{3}$ \\ Alexandros D. Tselepis, ${ }^{2}$ Dimitrios N. Kiortsis ${ }^{1}$ \\ ${ }^{1}$ Laboratory of Physiology, ${ }^{2}$ Laboratory of Biochemistry, Department of Chemistry, ${ }^{3}$ Department of Internal Medicine, \\ Medical School, University of Ioannina, Ioannina, Greece
}

\begin{abstract}
OBJECTIVE: The investigation of the relationship between high density lipoprotein (HDL) and adiponectin. DESIGN: Thirty-seven obese or overweight [body mass index $\geq 27 \mathrm{Kg} / \mathrm{m}^{2}$ ], hypertriglyceridemic patients underwent one of the following interventions for 3 months: 1) Low-calorie diet $(n=19), 2)$ Low-calorie diet plus fenofibrate $(n=18)$. RESULTS: Circulating total adiponectin did not change significantly in the low-calorie diet group. However, in the subgroup of patients whose high density lipoprotein cholesterol (HDL-C) decreased over the first month of diet, a statistically significant reduction of the circulating total adiponectin was observed $(p=0.010)$, while in the subgroup of patients whose HDL-C increased over the latter 2 months of the diet, an increase in circulating total adiponectin over the 2 months was found $(p=0.043)$. The percentage change of HDL-C over the first month of diet was positively correlated with the percentage change of circulating total adiponectin $(r=0.579, p=0.019)$. The percentage change of HDL-C over the 3 months of diet was positively correlated with the percentage changes of circulating total adiponectin $(r=0.527, p=0.030)$ and high molecular weight $(H M W)$ adiponectin $(r=0.524, p=0.031)$. The change in circulating total adiponectin over the first month of diet was positively correlated with the HDL-C at 1 month $(r=0.606$, $p=0.013$ ). The change in HMW adiponectin over the 3 months of diet was positively correlated with the HDL-C at 3 months $(r=0.602, p=0.011)$. The percentage change of circulating HMW adiponectin over the first month of fenofibrate treatment was positively correlated with the percentage change of HDL-C $(r=0.594, p=0.012)$. CONCLUSIONS: HDL is positively correlated with the changes in circulating adiponectin during dietary and fenofibrate treatment.

Key words: High density lipoprotein, Apolipoprotein A-I, Adiponectin, High molecular weight adiponectin, Diet, Fenofibrate.
\end{abstract}




\section{INTRODUCTION}

Adiponectin is an exclusive product of adipocytes. It augments insulin sensitivity and, in general, exerts a multitude of beneficial metabolic actions. ${ }^{1}$ Several factors affect the levels of circulating adiponectin, ${ }^{2}$ including obesity, in which plasma adiponectin levels are downregulated. ${ }^{3,4}$ Weight loss can induce metabolic changes, including the plasma levels of adipokines. ${ }^{5}$ With regard to the impact of weight loss on the levels of circulating adiponectin, there is considerable discrepancy in the literature. Specifically, some studies have demonstrated a rise of adiponectin levels during dietary interventions, ${ }^{6-13}$ whereas others could not confirm such changes. ${ }^{14-20}$ Therefore, changes in circulating adiponectin during dietary interventions may not be due to the weight loss per se but may represent a secondary phenomenon. The present study aimed to investigate the diet-induced changes of circulating adiponectin.

Adiponectin concentration in the blood has been found to be positively correlated with serum levels of high density lipoprotein cholesterol (HDL-C), independently of body mass index (BMI) and indices of insulin resistance, in a steady metabolic state. ${ }^{21}$ Notably, a previously published study has demonstrated that high density lipoprotein (HDL) may increase the expression of adiponectin in adipose tissue, ${ }^{22}$ indicating that changes in the circulating HDL may affect the expression of adiponectin and its secretion into the blood stream. However, there are few studies showing an association between the change in circulating adiponectin and changes in HDL-C during interventions that alter HDL-C levels, such as dietary ${ }^{4,14}$ and fibrate ${ }^{23}$ treatment. Moreover, these studies referred only to total adiponectin and not to high molecular weight (HMW) adiponectin, which is the most biologically active fraction of adiponectin. ${ }^{1}$ Furthermore, no reports exist on the relationship between changes in circulating adiponectin and HDL-C levels during brief short dietary interventions. This issue is of major significance since weight loss regiments result in an initial suppression of HDL-C followed by a subsequent elevation of its levels. ${ }^{24}$ The current study therefore attempted to elucidate whether HDL-C is the main determinant of plasma total adiponectin and HMW adiponectin levels, in a steady metabolic state and during short-term and medium-term interventions that change HDL-C, such as dietary and fenofibrate treatment. Another aim of the present study was to examine whether changes of circulating total adiponectin and HMW adiponectin correlate with changes in the BMI.

\section{MATERIALS AND METHODS}

\section{Subjects}

In the present study, 37 subjects ( 20 males and 17 females), aged 18-79, were recruited. Inclusion criteria were: $\mathrm{BMI} \geq 27 \mathrm{Kg} / \mathrm{m}^{2}$ and hypertriglyceridemia (Triglycerides (TRG) $\geq 150 \mathrm{mg} / \mathrm{dL}$ ). Exclusion criteria were: age less than 18 years old, kidney disease, liver disease and any endocrine disorder other than obesity or Type 2 diabetes mellitus, as well as change of body weight (BW) at more than $5 \%$ of the initial BW during the last 3 months and taking any of the following drugs within 3 weeks before the start of the study: hypolipidemic agents, antidiabetics, drugs for weight loss.

\section{Methodology}

Participants were randomly assigned to 2 schedules of medical intervention: 1) Hypocaloric low-fat diet $(n=19), 2)$ Hypocaloric low-fat diet with the concomitant oral administration of micronised fenofibrate (200 mg per day) $(n=18)$. Informed consent was obtained from all patients and the study was approved by the Ethics Committee of the University Hospital of Ioannina. All participants completed 4-day diet records at baseline, after 1 month and after 3 months of treatment. The treatment groups did not differ in their nutrient intake at baseline. A dietician calculated each patient's basal energy requirements and activity level and prescribed an individualized low-fat diet designed to bring about a 500 to $1000 \mathrm{kcal}$ reduction in daily energy intake. The administered diets consisted of a mean of $($ mean \pm SD) $1471 \pm 382 \mathrm{kcal} /$ day. The daily distribution of nutrients during the study was as follows: carbohydrates $52.4 \pm 3.5 \%$, fat $27.8 \pm 2.6 \%$ (monounsaturated $15.4 \pm 1.7 \%$, polyunsaturated $7.3 \pm 1.2 \%$ and saturated fatty acids $5.1 \pm 1.0 \%$ ) and protein $19.8 \pm 1.2 \%$. There were no differences in diet composition between the study groups. By the end of the 3-month period, the patients were consuming significantly fewer carbohydrates and saturated fatty acids as well as more monounsaturated fatty acids 
and n-3 polyunsaturated fatty acids compared with their baseline diet.

Plasma total adiponectin and HMW adiponectin were determined using the adiponectin multimeric enzyme immunoassay (ALPCO DIAGNOSTICS, Salem, NH, USA). To measure total adiponectin, samples were pretreated with Sample Pretreatment Buffer (Citrate buffer + SDS) which reduced multimeric adiponectin to dimmers. Subsequent measurement via ELISA quantified the amount of all multimers of adiponectin in the sample. To measure HMW adiponectin, samples were pretreated with Protease II, which selectively digested low molecular weight (LMW) adiponectin and mid-molecular weight (MMW) adiponectin. The remaining HMW fraction was treated with Sample Pretreatment Buffer which reduced these multimers to dimmers while also stopping the protease digestion. Subsequent measurement via ELISA quantified the amount of reduced HMW adiponectin but did not detect the digested LMW and MMW adiponectin.

In this ELISA, an antibody "sandwich" was utilized for detection of adiponectin. The microplate wells had been coated with an anti-human adiponectin monoclonal antibody and adiponectin in the standards and pretreated samples was captured during the first incubation. Washing removed all unbound material and a biotin-conjugated anti-human adiponectin monoclonal antibody bound to the immobilized adiponectin in the wells. After the second incubation and subsequent wash, step horseradish peroxidase (HRP)-labeled streptavidin was added. Following a third incubation and subsequent wash step, Ophenylenediamine (OPD) was added as substrate and the colorimetric reaction was terminated with the addition of diluted $\mathrm{H}_{2} \mathrm{SO}_{4}$. A dose response curve of absorbance unit (optical density at $450 \mathrm{~nm}$ ) vs. concentration was generated using the values obtained from standard. Adiponectin present in the patient samples was determined directly from this curve. The intra-assay coefficient of variation $(\mathrm{CV})$ was $5.4 \%$ for total adiponectin and 5\% for HMW adiponectin. The inter-assay $\mathrm{CV}$ was $5 \%$ for total adiponectin and $5.7 \%$ for HMW adiponectin. The HMW-to-total adiponectin ratio was calculated as the ratio of HMW adiponectin to total adiponectin. Total serum cholesterol (TC), HDL-C and TRG were measured by enzymatic methods using the BioMerieux reagents, as previously described. ${ }^{25}$ Serum apolipoprotein A-I (ApoA-I) was measured with a Behring Holding GmbH analyzer (Liederbach, Germany). The intra-assay CV was $0.72-0.91 \%$ for TC, 0.61-0.85\% for HDL-C and 2.7-3.4\% for ApoA-I. The inter-assay CV was 1.05-1.45\% for TC, 1.32-1.92 \% for HDL-C and 3.1-3.4\% for ApoA-I. Serum glucose concentration was measured via the enzymatic method and serum insulin was measured by radioimmunoassay. The homeostasis model assessment insulin resistance-index (HOMA-index), as a measure of insulin resistance, was calculated as [fasting insulin $(\mu \mathrm{U} / \mathrm{mL})] \mathrm{X}$ [fasting glucose $(\mathrm{mmol} / \mathrm{L})] / 22.5$.

All statistical analyses were performed using the SPSS 16.0 statistical package for Windows (SPSS Inc., 1989-2007). A Kolmogorov-Smirnov test was employed to verify the use of tests for normal distributed parameters. Normally distributed data are expressed as means \pm SD. Parameters with skewed distribution are reported as median (range). Repeated measures analysis of variance (Repeated ANOVA), independent-samples t test, Pearson's correlation analysis and multiple linear regression analysis were performed for normally distributed parameters, while the Friedman test and Spearman's correlation analysis were performed for non-normally distributed parameters. A two-tailed p value $<0.05$ was considered significant.

\section{RESULTS}

\section{Baseline}

The anthropometric and biochemical characteristics, at baseline, in the total of 38 patients are shown in Table 1. At baseline, plasma total adiponectin levels were positively correlated with HDL-C $(\mathrm{r}=0.375, \mathrm{p}=0.022)$, ApoA-I $(\mathrm{r}=0.522, \mathrm{p}=0.001)$ and age $(\mathrm{r}=0.417, \mathrm{p}=0.010)$ and tended to be negatively associated with HOMA-index $(r=-0.319, p=0.062)$. Similarly, circulating HMW adiponectin was positively correlated with HDL-C ( $\mathrm{r}=0.351, \mathrm{p}=0.033)$, ApoA-I $(\mathrm{r}=0.524, \mathrm{p}=0.001)$ and age $(\mathrm{r}=0.350, \mathrm{p}=0.034)$ and negatively correlated with HOMA-index $(\mathrm{r}=-0.371$, $\mathrm{p}=0.028)$. BMI did not correlate with circulating total adiponectin nor with HMW adiponectin. When ApoAI, HOMA-index and age were chosen as independent 
Table 1. The characteristics of the whole study population at baseline and their associations with circulating total adiponectin, circulating HMW adiponectin and HMW adiponectin proportion

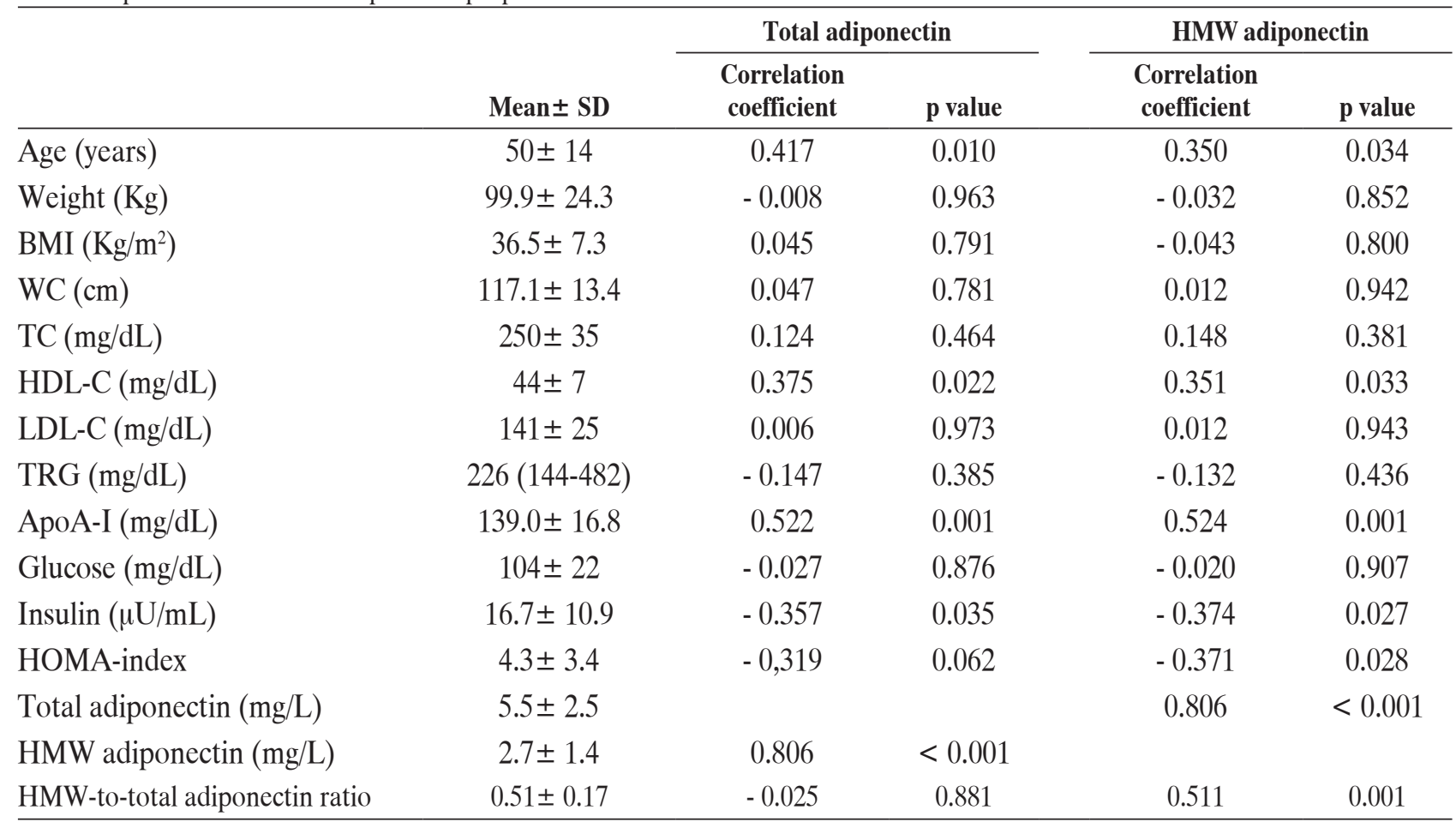

Data are means \pm SD for normally distributed variables or median (range) for non-normal variables.

BMI: body mass index; WC: waist circumference; TC: total cholesterol; HDL-C: high density lipoprotein-cholesterol; LDL-C: low density lipoprotein-cholesterol; TRG: triglycerides; ApoA-I: apolipopotein A-I; HOMA index: homeostasis model assessment insulin resistance-index.

variables in multiple linear regression analysis, only ApoA-I $(\beta=0.424$ and $p=0.019)$ was found to be a significant predictor of circulating HMW adiponectin $\left(\mathrm{R}^{2}=0.326\right.$ and $\mathrm{p}=0.006$ for the model $)$.

\section{Dietary intervention}

The characteristics of the subjects who followed the dietary treatment are shown in Table 2. The BMI decreased progressively over the 3 months of diet. Circulating total adiponectin and HMW adiponectin and HMW-to-total adiponectin ratio did not change significantly during diet (Figure 1). In the subgroup of patients whose HDL-C decreased over the first month of diet $(n=13)$, a decrease in circulating total adiponectin was found $(\mathrm{p}=0.010)$. In the subgroup of patients whose HDL-C did not decrease over the later 2 months of diet $(n=8)$, there was found an increase in circulating total adiponectin over the 2 months $(p=0.043)$ (data not shown).

The percentage change of circulating total adi- ponectin during the first month of diet was positively correlated with the percentage change of HDL-C $(\mathrm{r}=0.579, \mathrm{p}=0.019)$ and ApoA-I $(\mathrm{r}=0.565, \mathrm{p}=0.023)$ (Figure 2). The percentage change of circulating HMW adiponectin over the first month of diet was positively correlated with the percentage change of ApoA-I $(r=0.541, p=0.030)$ (Figure 2). The change in circulating total adiponectin over the first month of diet was positively correlated with the HDL-C $(\mathrm{r}=0.606, \mathrm{p}=0.013)$ and ApoA-I at 1 month $(\mathrm{r}=0.538$, $\mathrm{p}=0.032$ ) (Figure 2). No significant association between the percentage change of BMI over the first month of diet and the percentage changes of total adiponectin and HMW adiponectin was found.

Additionally, the percentage change of circulating total adiponectin over the 3 months of diet was positively correlated with the percentage change of HDL-C $(r=0.527, p=0.030)$ (Figure 3$)$ and tended to be negatively correlated with the percentage change of BMI $(r=-0.428, p=0.086)$. Moreover, the percent- 
Table 2. The characteristics of the patients $(n=19)$ that followed the dietary treatment at baseline, at 1 month and at 3 months

\begin{tabular}{lccc}
\hline & Baseline & 1 month & 3 months \\
\hline Age (years) & $46 \pm 15$ & & \\
Gender (males/ & $(12 / 7)$ & & \\
females) & & & \\
BMI $\left(\mathrm{Kg} / \mathrm{m}^{2}\right)$ & $36.4 \pm 5.9$ & $35.8 \pm 4.7^{\mathrm{a}}$ & $35.0 \pm 4.5^{\mathrm{b}, \mathrm{c}}$ \\
TC $(\mathrm{mg} / \mathrm{dL})$ & $245 \pm 28$ & $237 \pm 30$ & $226 \pm 29^{\mathrm{b}}$ \\
TRG (mg/dL) & 210 & 193 & 171 \\
& $(154-482)$ & $(94-331)$ & $(96-271)^{\mathrm{b}}$ \\
HDL-C (mg/dL) & $44 \pm 8$ & $43 \pm 8$ & $42 \pm 7$ \\
LDL-C (mg/dL) & $139 \pm 23$ & $132 \pm 21$ & $127 \pm 23$ \\
ApoA-I (mg/dL) & $137.3 \pm 19.8$ & $135.4 \pm 19.6$ & $126.1 \pm 25.1$ \\
HOMA-index & $4.0 \pm 2.4$ & $3.5 \pm 2.0$ & $2.8 \pm 1.6^{\mathrm{b}, \mathrm{c}}$ \\
Total adiponectin & $6.0 \pm 2.3$ & $5.7 \pm 2.9$ & $6.0 \pm 2.0$ \\
(mg/L) & & & \\
HMW adiponectin & $3.1 \pm 1.7$ & $3.0 \pm 1.8$ & $3.2 \pm 1.9$ \\
(mg/L) & & & \\
HMW-to-total & $0.50 \pm 0.15$ & $0.51 \pm 0.16$ & $0.51 \pm 0.14$ \\
adiponectin ratio & & & \\
\hline Datare means & & &
\end{tabular}

Data are means \pm SD for normally distributed variables or median (range) for non-normal variables.

BMI: body mass index; TC: total cholesterol; TRG: triglycerides; HDL-C: high density lipoprotein-cholesterol; LDL-C: low density lipoprotein-cholesterol; ApoA-I: apolipopotein A-I; HOMA index: homeostasis model assessment insulin resistance-index. ${ }^{\mathrm{a}} \mathrm{p}<0.050$ for 1 month vs baseline; ${ }^{\mathrm{b}} \mathrm{p}<0.050$ for 3 months vs baseline; ${ }^{\mathrm{p}} \mathrm{p}<0.050$ for 3 months vs 1 month.

age change of HMW adiponectin over the 3 months of diet was positively correlated with the percentage changes of HDL-C $(r=0.524, p=0.031)$ and ApoA-I $(\mathrm{r}=0.517, \mathrm{p}=0.034)$ (Figure 3$)$. The change in HMW adiponectin over the 3 months of diet was positively correlated with the HDL-C at 3 months $(r=0.602$, $\mathrm{p}=0.011)$ (Figure 3).

\section{Fenofibrate treatment}

The characteristics of the subjects who followed the fenofibrate treatment are shown in Table 3. Circulating total adiponectin, HMW adiponectin and HMW-to-total adiponectin ratio did not change significantly during fenofibrate treatment (Figure 1). The percentage change of HDL-C over the first month of fenofibrate treatment was positively correlated with the percentage changes of circulating HMW adiponectin $(r=0.594, p=0.012)$ and HMW-to-total adiponectin ratio $(\mathrm{r}=0.505, \mathrm{p}=0.039)$. Moreover, the ratio (change of total adiponectin over the first month/HDL-C at 1 month) did not differ significantly between dietary and fenofibrate treatment. A similar result was found regarding HMW adiponectin.

\section{DISCUSSION}

The findings of the present study showed that, at baseline, in the whole study population, circulating total adiponectin and HMW adiponectin correlated with HDL-C, ApoA-I and HOMA-index and not with BMI. Indeed, the correlation coefficient between adiponectin levels and BMI was approximately zero. The current study included subjects with increased BMI. It has consistently been shown that the inverse relationship between circulating total adiponectin and BMI is present only in nonobese subjects, whereas this association is absent in obese patients. ${ }^{4}$ Moreover, multiple regression analysis revealed that the best predictor of HMW adiponectin was ApoA-I. These findings raise the possibility that the ApoA-Icontaining lipoprotein HDL is the major determinant of circulating total adiponectin and HMW adiponectin, at least in a steady metabolic state.

In the current study, fairly uniform associations were found between the percentage change of HDL$\mathrm{C}$ and the percentage changes of circulating total adiponectin and HMW adiponectin, in both dietary and fenofibrate treatment groups. Moreover, these associations were present during the first month of the dietary regiment as well as during the 3 months. The studies showing an association between the change in circulating adiponectin and the change in HDL-C during dietary ${ }^{4,14}$ and fenofibrate ${ }^{23}$ treatment applied only to total adiponectin and not to HMW adiponectin, which is the most biologically active fraction of adiponectin. ${ }^{1}$ Further, there has been as yet no investigation as to whether the association between the diet-induced change in HDL-C and the change in circulating adiponectin is present during short-term weight loss, as has however been undertaken in the present study. This issue is of major importance since weight loss treatment has different effects on HDL-C, causing a decrease in HDL-C during short-term diets and an increase in HDL-C during long-term diets. ${ }^{24}$ Notably, in the current study, although BMI decreased over the first month of diet, 


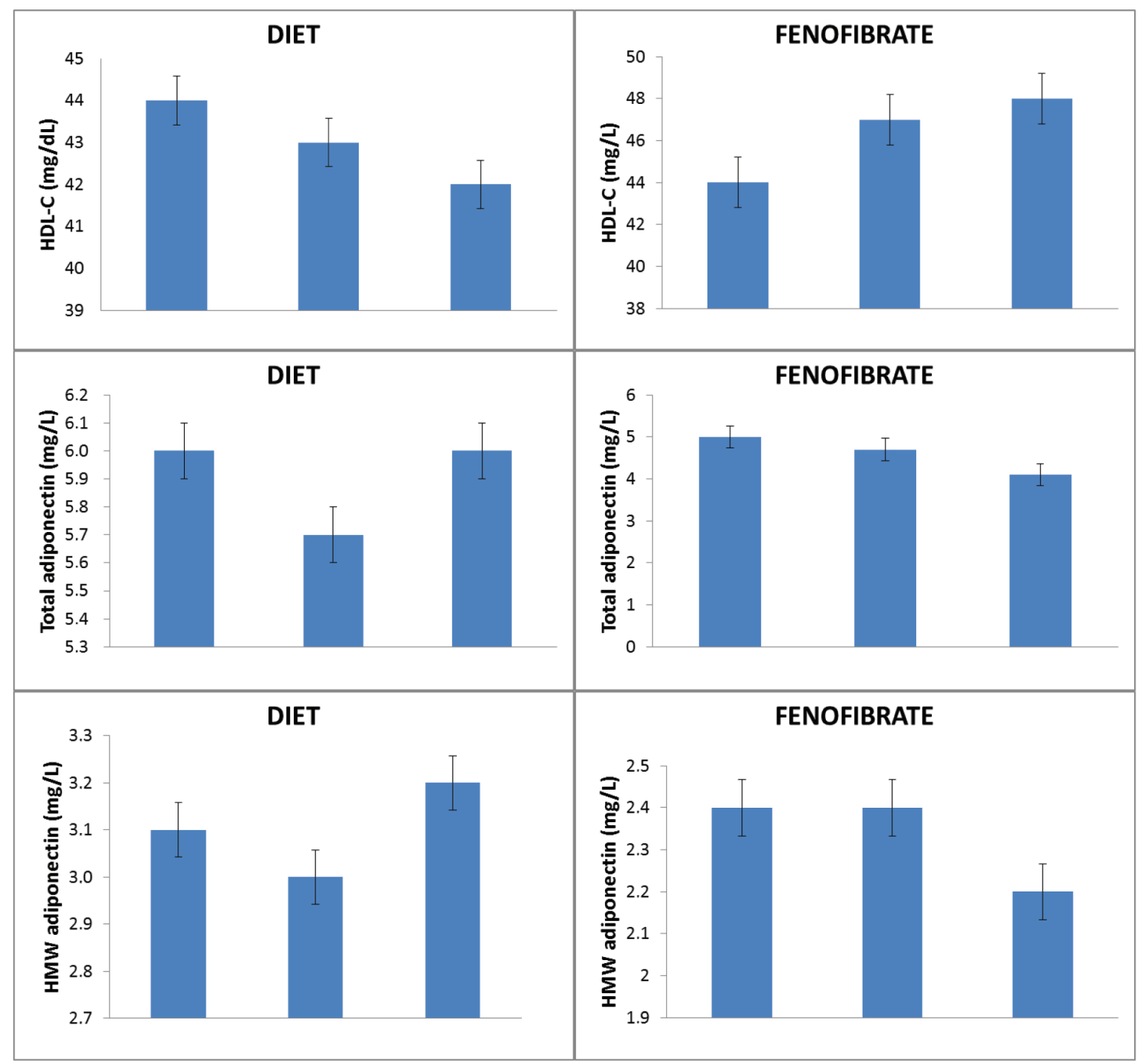

Figure 1. Six schematic representations show the changes in HDL-C, total adiponectin and HMW adiponectin during dietary and fenofibrate treatments. All these changes were statistically nonsignificant.

the percentage change of circulating total adiponectin and HMW adiponectin did not correlate significantly with the percentage change of BMI. No other studies have demonstrated that the association between the diet-induced changes of circulating adiponectin and HDL-C exists, even in the absence of association between the changes in circulating adiponectin and BMI. This finding raises the possibility that the association between adiponectin and HDL is independent of BMI. It has previously consistently been reported that the association between the diet-induced change in HDL-C and circulating total adiponectin is independent of the change in BMI during a dietary treatment of 6-12 months. ${ }^{4}$ Similarly, in the present study the percentage changes of total adiponectin and HMW adiponectin over the 3 months of diet correlated with the percentage change of HDL-C, whereas the associations with the percentage change of BMI were marginally nonsignificant and were noted only for total adiponectin and not for HMW adiponectin.

It is also of note that in the present study, although circulating total adiponectin did not change significantly in the whole study population during diet, a change in circulating total adiponectin was found only 

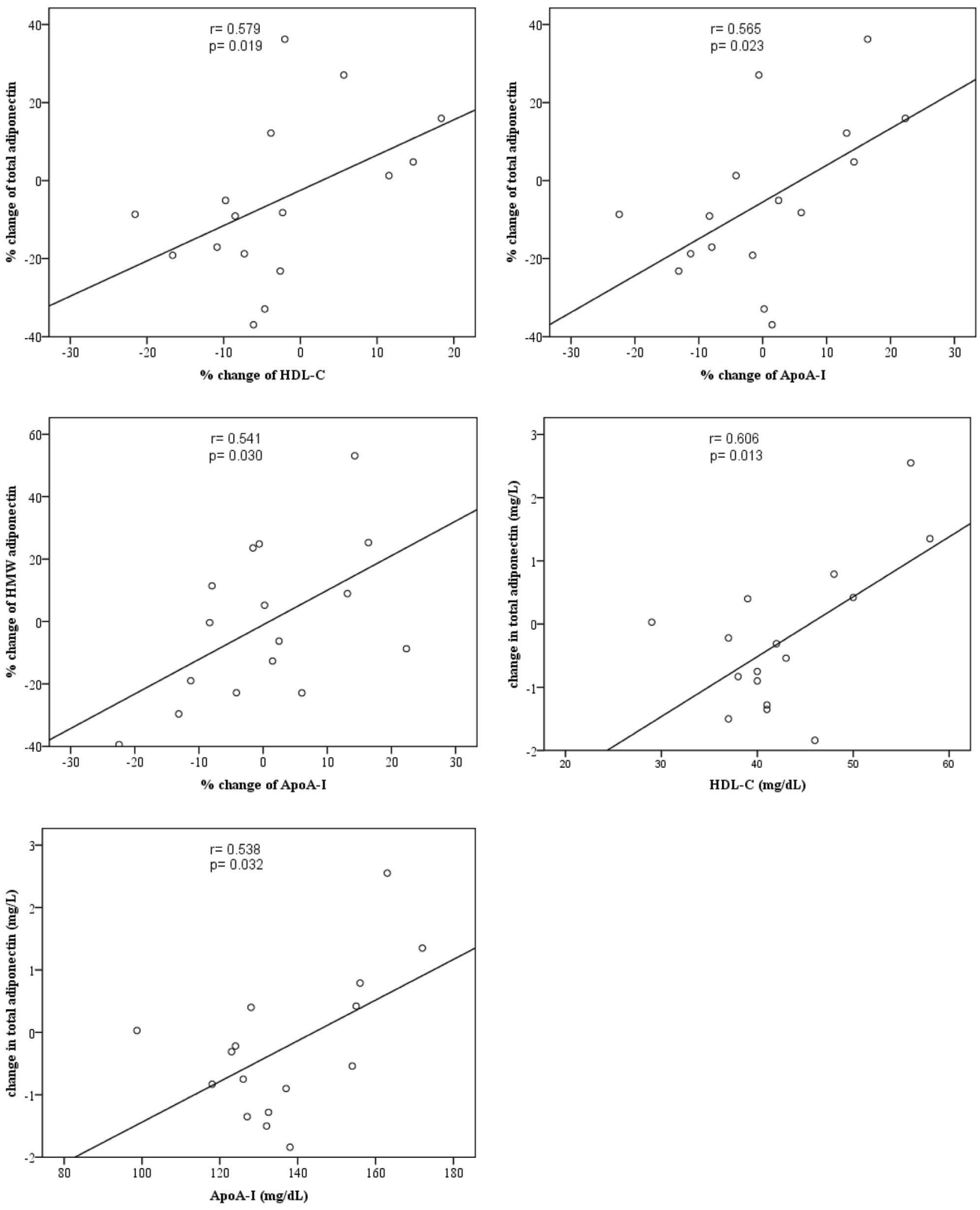

Figure 2. Dietary treatment. Relationship between the percentage change of circulating total adiponectin over the first month and the percentage changes of high density lipoprotein-cholesterol (HDL-C) and Apolipopotein A-I (ApoA-I). Association between the percentage change of circulating high molecular weight (HMW) adiponectin over the first month and the percentage change of ApoA-I. Relationship between the change in circulating total adiponectin over the first month and HDL-C, ApoA-I at 1 month. 

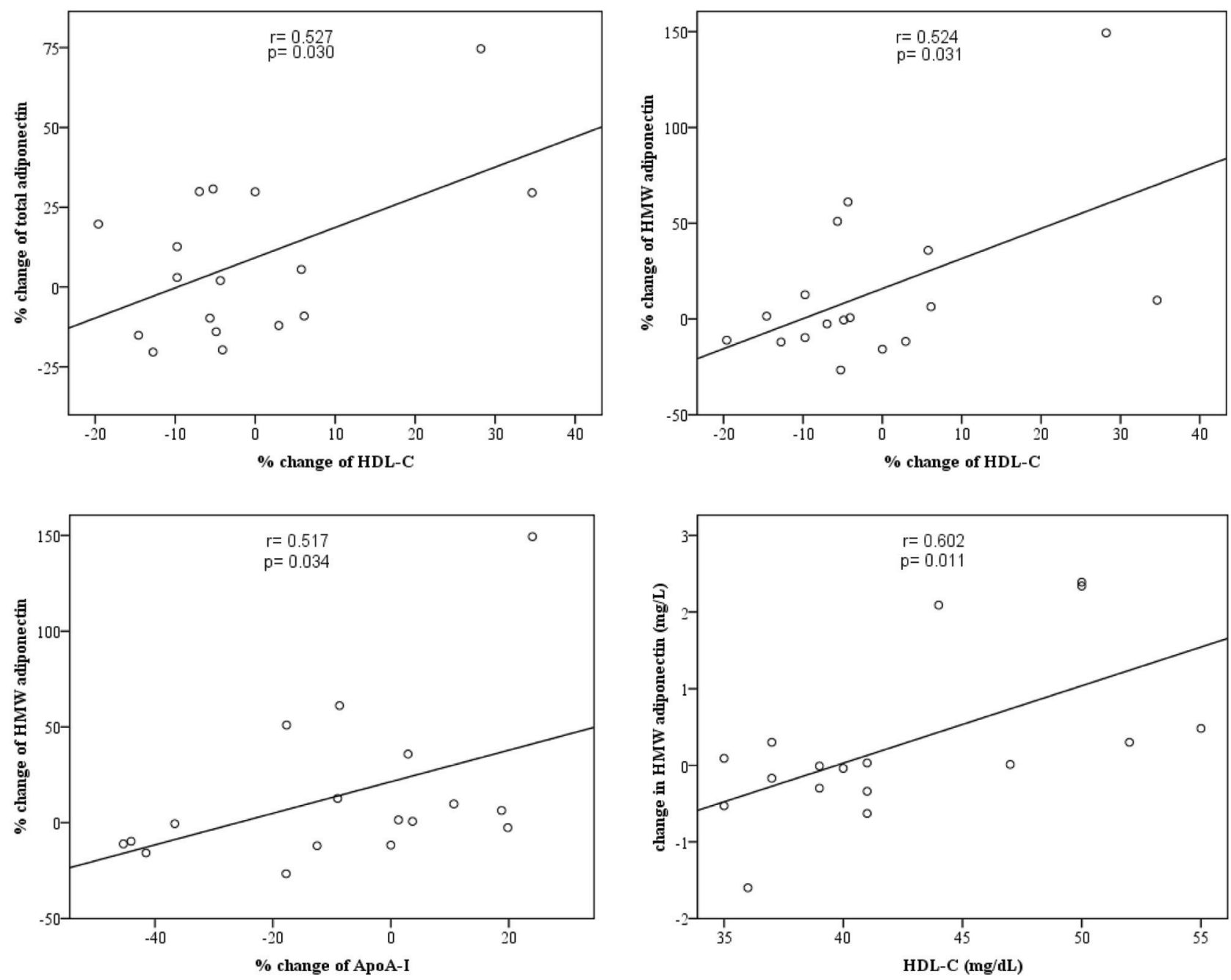

Figure 3. Dietary treatment. Relationship between the percentage change of circulating total adiponectin and HMW adiponectin over the 3 months and the percentage change of HDL-C. Association between the percentage change of HMW adiponectin over the 3 months and the percentage change of ApoA-I. Relationship between the change in circulating HMW adiponectin over the 3 months and the HDL-C at 3 months.

in the subgroup of patients whose HDL-C changed. This finding may explain the fact that in some studies, diet did not cause significant change in circulating adiponectin, indicating that circulating adiponectin may not be influenced by weight loss per se and is possibly affected by the changes in HDL-C. Moreover, in the current study the HDL-C at any time point of the dietary intervention was strongly associated with the relevant changes in circulating total adiponectin and HMW adiponectin from baseline. This is the first time such an association has been reported, indicating that the value of HDL-C influences the change rate of circulating total adiponectin and HMW adiponectin. Specifically, the diet-induced change in circulating adiponectin has so far been associated only with the change in HDL-C $\mathrm{C}^{14}$ and not with the value of HDL-C. In this respect, HDL-C appears to be linked with the metabolic pathway which is responsible for the diet-induced changes in circulating total adiponectin and HMW adiponectin. Even more importantly, the ratio (change of total adiponectin or HMW adiponectin over the first month/HDL-C at 1 month) did not differ between dietary and fenofibrate treatment. Thus, the magnitude of impact of HDL on circulating total adiponectin and HMW adiponectin appears to be similar between dietary and fenofibrate treatment, implying that the changes in adiponectin are caused by HDL per se, independently of the ap- 
Table 3. The characteristics of the patients $(n=18)$ that followed the fenofibrate treatment at baseline, at 1 month and at 3 months

\begin{tabular}{lccc}
\hline & Baseline & 1 month & 3 months \\
\hline Age (years) & $53 \pm 13$ & & \\
Gender (males/ & $(8 / 10)$ & & \\
females) & & & \\
BMI $\left(\mathrm{Kg} / \mathrm{m}^{2}\right)$ & $36.6 \pm 8.7$ & $36.0 \pm 8.9^{\mathrm{a}}$ & $35.0 \pm 9.1^{\mathrm{b}, \mathrm{c}}$ \\
TC $(\mathrm{mg} / \mathrm{dL})$ & $255 \pm 42$ & $222 \pm 33^{\mathrm{a}}$ & $227 \pm 37^{\mathrm{b}}$ \\
TRG $(\mathrm{mg} / \mathrm{dL})$ & 295 & 139 & 142 \\
& $(144-400)$ & $(105-383)^{\mathrm{a}}$ & $(89-368)^{\mathrm{b}}$ \\
HDL-C (mg/dL) & $44 \pm 7$ & $47 \pm 8$ & $48 \pm 7$ \\
LDL-C (mg/dL) & $142 \pm 28$ & $120 \pm 22^{\mathrm{a}}$ & $125 \pm 21^{\mathrm{b}}$ \\
ApoA-I (mg/dL) & $140.6 \pm 14.1$ & $144.2 \pm 22.3$ & $148.4 \pm 16.9$ \\
HOMA-index & $4.6 \pm 4.3$ & $3.5 \pm 1.6$ & $2.7 \pm 1.5^{\mathrm{c}}$ \\
Total adiponectin & $5.0 \pm 2.7$ & $4.7 \pm 2.3$ & $4.1 \pm 1.4$ \\
(mg/L) & & & \\
HMW adiponectin & $2.4 \pm 1.1$ & $2.4 \pm 1.2$ & $2.2 \pm 0.9$ \\
(mg/L) & & & \\
HMW-to-total & $0.52 \pm 0.20$ & $0.53 \pm 0.20$ & $0.54 \pm 0.20$ \\
adiponectin ratio & & & \\
\hline Data a means & & &
\end{tabular}

Data are means \pm SD for normally distributed variables or median (range) for non-normal variables.

BMI: body mass index; TC: total cholesterol; TRG: triglycerides; HDL-C: high density lipoprotein-cholesterol; LDL-C: low density lipoprotein-cholesterol; ApoA-I: apolipopotein A-I; HOMA index: homeostasis model assessment insulin resistance-index. ${ }^{\mathrm{a}} \mathrm{p}<0.050$ for 1 month vs baseline; ${ }^{\mathrm{b}} \mathrm{p}<0.050$ for 3 months vs baseline; ${ }^{\mathrm{p}}<0.050$ for 3 months vs 1 month.

plied treatment. Notably, a previous study mentioned that incubation of adipocytes with HDL upregulated adiponectin expression. ${ }^{22}$ Moreover, this study reported that ApoA-I gene transfer in mice resulted in an increase in HDL-C, which was paralleled by an increase in plasma adiponectin levels and adiponectin expression in abdominal fat. HDL-C therefore appears to be a causal factor of the changes in total adiponectin and HMW adiponectin rather than a factor that solely reflects the metabolism of adiponectin. In this context, given the well-known association of circulating adiponectin with various cardiometabolic parameters, adiponectin may constitute the mediator of some of the cardiometabolic actions of HDL. It has consistently been reported that the association between circulating adiponectin and coronary heart disease is attenuated after adjustment for HDL-C. ${ }^{26}$
Furthermore, it has been shown that reconstituted HDL reduces plasma glucose in patients with type 2 diabetes mellitus by increasing plasma insulin and activating AMP-activated protein kinase in skeletal muscle. ${ }^{27}$ It is well known that the insulin sensitizing effects of adiponectin are mediated via the activation of AMP-activated protein kinase in skeletal muscle. ${ }^{1}$ Therefore, adiponectin may constitute the mediator of the insulin sensitizing effects of HDL.

The concept of the HDL-induced changes in circulating adiponectin can adequately explain the diet-induced changes in circulating adiponectin which have been reported so far. Specifically, it is well known that HDL-C decreases during periods of active weight loss, whereas HDL-C increases during periods of stabilized weight loss compared to baseline values..$^{24}$ Based on this point of view, weight loss studies lasting no more than 6 weeks (mostly active weight loss periods) reported a decrease in HDL-C, whereas weight loss studies lasting for longer periods (mostly stabilized weight loss periods) showed an increase in HDL-C. ${ }^{24}$ Regarding the impact of diet on circulating adiponectin, long-term diets (lasting at least 5 months) induce an increase in circulating adiponectin, ${ }^{6-13}$ diets of very short duration (lasting no more than 1 month) cause a decrease in circulating adiponectin ${ }^{20}$ and diets of intermediate duration did not have any significant impact on circulating adiponectin. ${ }^{14-19}$ Therefore, the decrease in HDL-C during short-term diets may induce the decrease in circulating adiponectin, whereas the increase in HDL-C during long-term diets possibly provokes the increase in circulating adiponectin. Additionally, it has been shown that exercise can increase circulating adiponectin without any significant change in BMI. ${ }^{28,29}$ A plausible explanation for this phenomenon is that the exercise-induced increase in HDL-C may cause the increase in plasma adiponectin levels.

In conclusion, HDL-C is positively correlated with circulating total adiponectin and HMW adiponectin in a steady metabolic state as well as during medical interventions that affect HDL-C, such as dietary and fibrate treatment. This regulation appears to exist independently of any change of the BMI. In this respect, adiponectin may constitute the mediator of some of the beneficial cardiometabolic actions of 
HDL. Further studies are needed in more diverse populations and with various treatments to support the abovementioned results.

\section{The study was partially funded by the Hellenic Endocrine Society.}

The authors have no conflict of interest.

\section{REFERENCES}

1. Guerre-Millo M, 2008 Adiponectin: an update Diabetes \& Metabolism 34: 12-18.

2. Evagelidou EN, Giapros VI, Challa AS, Kiortsis DN, Tsatsoulis AA, Andronikou SK, 2007 Serum adiponectin levels, insulin resistance, and lipid profile in children born small for gestational age are affected by the severity of growth retardation at birth. Eur J Endocrinol. 156: 271-277.

3. Arita Y, Kihara S, Ouchi N et al, 1999 Paradoxical decrease of an adipose-specific protein, adiponectin, in obesity. Biochem Biophys Res Commun. 257: 79-83.

4. Baratta R, Amato S, Degano C, et al, 2004 Adiponectin relationship with lipid metabolism is independent of body fat mass: evidence from both cross-sectional and intervention studies. J Clin Endocrinol Metab 89: 2665-2671.

5. Kiortsis DN, Tzotzas T, Giral P, et al, 2001 Changes in lipoprotein(a) levels and hormonal correlations during a weight reduction program. Nutr Metab Cardiovasc Dis 11: 153-157.

6. Yang WS, Lee WJ, Funahashi T, et al, 2001 Weight reduction increases plasma levels of an adipose-derived anti-inflammatory protein, adiponectin. J Clin Endocrinol Metab 86: 3815-3819.

7. Esposito K, Pontillo A, Di Palo C, et al, 2003 Effect of weight loss and lifestyle changes on vascular inflammatory markers in obese women: a randomized trial. JAMA 289: 1799-1804.

8. Al-Sarraj T, Saadi H, Calle MC, Volek JS, Fernandez ML, 2009 Carbohydrate restriction, as a first-line dietary intervention, effectively reduces biomarkers of metabolic syndrome in Emirati adults. J Nutr 139: 1667-1676.

9. Faraj M, Havel PJ, Phélis S, Blank D, Sniderman AD, Cianflone K, 2003 Plasma acylation-stimulating protein, adiponectin, leptin, and ghrelin before and after weight loss induced by gastric bypass surgery in morbidly obese subjects. J Clin Endocrinol Metab 88: 1594-1602.

10. Kopp HP, Krzyzanowska K, Möhlig M, Spranger J, Pfeiffer AF, Schernthaner G, 2005 Effects of marked weight loss on plasma levels of adiponectin, markers of chronic subclinical inflammation and insulin resistance in morbidly obese women. Int J Obes (Lond) 29: 766-771.

11. Lazzer S, Vermorel M, Montaurier C, Meyer M, Boirie Y, 2005 Changes in adipocyte hormones and lipid oxidation associated with weight loss and regain in severely obese adolescents. Int J Obes (Lond) 29: 1184-1191.
12. Bobbert T, Rochlitz H, Wegewitz U, et al, 2005 Changes of adiponectin oligomer composition by moderate weight reduction. Diabetes 54: 2712-2719.

13. Manco M, Fernandez-Real JM, Equitani F, et al, 2007 Effect of massive weight loss on inflammatory adipocytokines and the innate immune system in morbidly obese women. J Clin Endocrinol Metab 92: 483-490.

14. Keogh JB, Brinkworth GD, Clifton PM, 2007 Effects of weight loss on a low-carbohydrate diet on flow-mediated dilatation, adhesion molecules and adiponectin. Br J Nutr 98: 852-859.

15. Xydakis AM, Case CC, Jones PH, et al, 2004 Adiponectin, inflammation, and the expression of the metabolic syndrome in obese individuals: the impact of rapid weight loss through caloric restriction. J Clin Endocrinol Metab 89: 2697-2703.

16. Keogh JB, Brinkworth GD, Noakes M, Belobrajdic DP, Buckley JD, Clifton PM, 2008 Effects of weight loss from a very-low-carbohydrate diet on endothelial function and markers of cardiovascular disease risk in subjects with abdominal obesity. Am J Clin Nutr 87: 567-576.

17. Arvidsson E, Viguerie N, Andersson I, Verdich C, Langin D, Arner P, 2004 Effects of different hypocaloric diets on protein secretion from adipose tissue of obese women. Diabetes 53: 1966-1971.

18. Polak J, Kovacova Z, Holst C, et al, 2008 Total adiponectin and adiponectin multimeric complexes in relation to weight loss-induced improvements in insulin sensitivity in obese women: the NUGENOB study. Eur J Endocrinol 158: 533-541.

19. Dvoráková-Lorenzová A, Suchánek P, Havel PJ, et al, 2006 The decrease in C-reactive protein concentration after diet and physical activity induced weight reduction is associated with changes in plasma lipids, but not interleukin-6 or adiponectin. Metabolism 55: 359-365.

20. Lejeune MP, Hukshorn CJ, Saris WH, Westerterp-Plantenga MS, 2007 Effects of very low calorie diet induced body weight loss with or without human pegylated recombinant leptin treatment on changes in ghrelin and adiponectin concentrations. Physiol Behav 91: 274-280.

21. Kazumi T, Kawaguchi A, Hirano T, Yoshino G, 2004 Serum adiponectin is associated with high-density lipoprotein cholesterol, triglycerides, and low-density lipoprotein particle size in young healthy men. Metabolism 53: 589-593.

22. Van Linthout S, Foryst-Ludwig A, Spillmann F, et al, 2010 Impact of HDL on adipose tissue metabolism and adiponectin expression. Atherosclerosis 210: 438-444.

23. Koh KK, Quon MJ, Lim S, et al, 2011 Effects of fenofibrate therapy on circulating adipocytokines in patients with primary hypertriglyceridemia. Atherosclerosis 214: 144-147.

24. Dattilo AM, Kris-Etherton PM, 1992 Effects of weight reduction on blood lipids and lipoproteins: a metaanalysis. Am J Clin Nutr 56: 320-328.

25. Christou GA, Tellis CC, Elisaf MS, Tselepis AD, Kiortsis 
DN, 2011 The Changes in Plasma Retinol-Binding Protein 4 Levels are Associated With Those of the Apolipoprotein B-Containing Lipoproteins During Dietary and Drug Treatment. Angiology. (In Press) (Epub ahead of print). 26. Schulze MB, Shai I, Rimm EB, Li T, Rifai N, Hu FB. 2005 Adiponectin and future coronary heart disease events among men with type 2 diabetes. Diabetes 54: 534-539.

27. Drew BG, Duffy SJ, Formosa MF, et al, 2009 Highdensity lipoprotein modulates glucose metabolism in patients with type 2 diabetes mellitus. Circulation 119:
2103-2111

28. Kriketos AD, Gan SK, Poynten AM, Furler SM, Chisholm DJ, Campbell LV, 2004 Exercise increases adiponectin levels and insulin sensitivity in humans. Diabetes Care 27: 629-630.

29. Balagopal P, George D, Yarandi H, Funanage V, Bayne E, 2005 Reversal of obesity-related hypoadiponectinemia by lifestyle intervention: a controlled, randomized study in obese adolescents. J Clin Endocrinol Metab 90: 6192-6197. 